\title{
Species-specific urokinase receptor ligands reduce glioma growth and increase survival primarily by an antiangiogenesis mechanism
}

\author{
Xingyao $\mathrm{Bu}^{1}$, Vazgen Khankaldyyan ${ }^{1}$, Ignacio Gonzales-Gomez ${ }^{1}$, Susan Groshen ${ }^{2}$, \\ Wei Ye ${ }^{2}$, Shaoqiu Zhuo ${ }^{3, *}$, Jaume Pons ${ }^{3, \dagger}$, Jennifer R Stratton ${ }^{3, \dagger}$, Steven Rosenberg ${ }^{3, \ddagger}$ \\ and Walter E Laug ${ }^{1}$

\begin{abstract}
${ }^{1}$ Department of Pediatrics; ${ }^{2}$ Department of Preventive Medicine, Childrens Center for Cancer and Blood Diseases, Childrens Hospital Los Angeles, Los Angeles, CA, USA and ${ }^{3}$ USC Keck School of Medicine and Chiron Corporation, Emeryville, CA, USA
\end{abstract}

\begin{abstract}
Species-specific urokinase receptor (UPAR) ligands with improved pharmacokinetics were generated by sitespecific mutagenesis and amino-terminal pegylation. These molecules were used to probe the role of uPAR in brain tumor progression and angiogenesis. The ligands blocked endothelial cell tube formation in Matrigel in a species-specific manner and reduced both baseline and uPA amino-terminal fragment-stimulated cell migration on vitronectin gradients. Treatment of U87MG gliomas implanted orthotopically in mice with single speciesspecific or combination uPAR ligands resulted in significant decreases in tumor size, which translated to increases in survival time, and which were most significant when the murine-specific ligand was included. Further analysis of tumors showed that the reduced sizes were correlated with a decrease in tumor cell proliferation and mean vessel density and an increase in tumor cell apoptosis. In addition, a large increase in collagen deposition was observed in the treated groups. Statistical analysis showed that the combination therapy demonstrated a clear synergy as compared to the individual agent treatments. These results suggest that the major role of the UPAR system in brain tumor progression is in the stromal compartment and particularly in neovascularization, a hallmark of invasive brain tumors.
\end{abstract}

Laboratory Investigation (2004) 84, 667-678, advance online publication, 19 April 2004; doi:10.1038/labinvest.3700089

Keywords: urokinase receptor; brain tumor; angiogenesis; proteolysis; adhesion

The urokinase-type plasminogen activator (uPA) system has been implicated in cancer progression, invasion, and angiogenesis as well as tumor prognosis. ${ }^{1-5}$ This system is central to cell-associated proteolytic activity as well as cell migration and adhesion, through interactions between the urokinase receptor (UPAR) and integrins, as well as extracellular matrix components, especially vitronectin. ${ }^{6,7}$ Several in vivo studies have indicated that modulation of the expression of UPAR or blockade of

Correspondence: W Laug, MD, Childrens Hospital Los Angeles, 4650 Sunset Blvd, MS \#57, Los Angeles, CA 90027, USA.

E-mail: wlaug@chla.usc.edu

*Current address: Diadexus, Inc., 343 Oyster Point Blvd, S. San Francisco, CA 94080, USA.

Current address: Rinat Neuroscience, 3155 Porter Drive, Palo Alto, CA 94304, USA.

${ }^{\ddagger}$ Current address: XDx, Inc., 750 Gateway Blvd, Suite H, S. San Francisco, CA 94080, USA.

Received 21 November 2003; revised and accepted 21 January 2004; published online 19 April 2004
uPAR with catalytically inactive uPA fragments lead to reductions in tumor growth, tumor angiogenesis, and an increase in tumor dormancy in a variety of tumor types., ${ }^{3,8-11}$ In addition, studies of human tumors have shown that different components of the uPA system are expressed by both transformed epithelial cells and stromal cells. ${ }^{12,13}$ Finally, studies using uPA knock-out mice have shown a direct role for stromal uPA in tumor progression in some systems, ${ }^{14,15}$ although uPAR blockade in the absence of uPA has also shown in vivo effects. ${ }^{16}$

Brain tumors, and specifically gliomas, have been shown to overexpress components of the uPA system, and tumor grade has been shown to correlate with expression levels. ${ }^{17}$ Reduction in levels of UPAR expression using antisense technology has been shown to decrease tumor growth in an in vivo model..$^{18}$ In addition, gliomas are reported to be one of the most highly vascularized tumors, suggesting that antiangiogenic approaches to these aggressive tumors might be useful. In this study, we have taken advantage of the species specificity of the 
uPA:uPAR interaction ${ }^{19}$ to probe the role of both human tumor and murine stromal uPAR in tumor growth and angiogenesis in an orthotopic human xenograft model of glioma in immunodeficient mice. The results suggest that stromal uPAR, especially on endothelial cells, plays a predominant role in glioma tumor growth, and blockade of murine stromal UPAR in this setting, increases survival, decreases tumor growth and tumor neovascularization, and increases tumor cell apoptosis. These results show the importance of the stromal cell uPA system contribution to brain tumor progression.

\section{Materials and methods}

\section{Materials}

Human uPA1-48 (h1-48) was expressed in recombinant yeast as described previously. ${ }^{20}$ The quadruple mutant of h1-48 with high affinity for the murine uPAR (hm1-48, huPA1-48, N22Y, N27R, H29R, W30R) was expressed in the same system. ${ }^{8}$ Peptides were purified from yeast cell-conditioned media by S-Fractogel ion exchange chromatography. Specific amino-terminal pegylation of these peptides was performed by the method of Gaertner and Offord..$^{21}$ Specifically, purified huPA1-48 and the mutant protein were concentrated to $7-10 \mathrm{mg} / \mathrm{ml}$ by diafiltration, the $\mathrm{pH}$ was adjusted to 6.8 , and three molar equivalents of freshly prepared sodium metaperiodate were added. The reaction was incubated for $30 \mathrm{~min}$ at room temperature with stirring and protected from light and was then quenched by the addition of sodium acetate to a final $\mathrm{pH}$ of 4.5. After dialysis against $30 \mathrm{mM}$ sodium acetate, $\mathrm{pH} 4.5$, the pegylation reaction was performed using a 3-5-fold molar excess of methoxy-PEG-hydrazide $20000 \mathrm{MW}$ (Shearwater Polymers, Huntsville, AL, USA) at $37^{\circ} \mathrm{C}$ for $30 \mathrm{~h}$ with gentle stirring and protected from light. The pegylated proteins were purified by Fractogel cation exchange chromatography, diafiltered against a $10000 \mathrm{MW}$ Amicon filter, and any contaminating endotoxin removed by passage through a TMAE Fractogel column equilibrated with phosphatebuffered saline (PBS). The resulting purified pegylated peptides were analyzed by matrix-assisted laser desorption mass spectroscopy, SDS-PAGE, human and murine uPAR radio-receptor-binding assays, surface plasmon resonance (BIACore), and amino-acid analysis. In all cases, the resulting proteins showed a molecular mass centered around $27 \mathrm{kDa}$, endotoxin of $<0.5 \mathrm{EU} / \mathrm{mg}$ (LAL assay), and $>95 \%$ purity by SDS-PAGE.

Antibodies used for FACS analysis and immunohistochemistry were monoclonal mouse anti-human uPAR (CD 87) antibody 3936 (American Diagnostica, Greenwich, CT, USA), polyclonal rabbit anti-mouse uPAR antibody (Chiron), rat anti-mouse CD31 monoclonal antibody (MEC 13.3, BD Biosciences, San Diego, CA, USA), and monoclonal mouse antihuman Ki-67 (DAKO, Carpinteria, CA, USA). Vitro- nectin was purchased from Promega (Madison, MI, USA) and Matrigel from BD Biosciences (San Diego, CA, USA). Human ATF was purchased from American Diagnostica.

\section{Cell Line}

The human brain tumor cell line U87MG (glioblastoma) was purchased from ATCC, VA, USA and grown in RPMI medium with $10 \%$ fetal bovine serum in $5 \% \mathrm{CO}_{2}$ at $37^{\circ} \mathrm{C}$. Primary human and mouse brain capillary endothelial cells (BCEC) were isolated, characterized, and grown as described ${ }^{22}$ and used between passages 5 and 12 .

\section{Pharmacokinetics}

The in vivo half-lives of uPA1-48 and PEGh1-48 were determined after intravenous (i.v.) injection in mice. Proteins were injected at $10 \mathrm{mg} / \mathrm{kg}$ and blood samples were collected at various time points and the amount of human uPAR inhibitory activity was measured using a UPAR binding assay as previously described, but in the presence of a constant amount $(50 \%)$ of mouse plasma. ${ }^{23}$ The half-life of uPA1-48 was determined to be less than $10 \mathrm{~min}$ whereas PEGh1-48 had a half-life of $130 \mathrm{~min}$. Subsequent experiments demonstrated that PEGh1-48 was very efficiently absorbed after subcutaneous injection, as well.

\section{Brain Tumors}

Details of the xenotransplant model in nu/nu mice have been described previously. ${ }^{24}$ In brief, $10^{6}$ tumor cells suspended in $10 \mu \mathrm{l}$ serum-free RPMI medium were injected over $10 \mathrm{~min}$ into the forebrain at $1.5 \mathrm{~mm}$ lateral and $0.5 \mathrm{~mm}$ anterior to the Bregma, at a depth of $2.5 \mathrm{~mm}$. The burr hole was closed with bone wax. Mice were kept under general anesthesia during the procedure with administration of ketamine $(100 \mathrm{mg} / \mathrm{kg})$ and xylazine $(10 \mathrm{mg} / \mathrm{kg})$. Subcutaneous administration of mouse or human pegylated uPA1-48 was twice weekly at $300 \mu \mathrm{g} / 100 \mu \mathrm{l}$ when given alone or at $100 \mu \mathrm{g} / 100 \mu \mathrm{l}$ each when given combined. The weight of animals was monitored twice weekly and mice were killed when showing signs of tumor progression such as cachexia, hunchback presentation, or any signs of paralysis. The remaining mice were killed when drug supply became limiting. Tumor size is given in millimeters and represents the largest diameter on serial sections of the tumor-containing brain area.

For histological studies, brains of killed animals were immediately removed and either snap frozen in liquid nitrogen for immunofluorescence studies or fixed in $10 \%$ buffered formalin and embedded in paraffin for hematoxylin and eosin (H\&E) and Masson's Trichrome staining and immunohisto- 
chemistry. Tumor size was determined. Animal studies were carried out according to the NIH guidelines and approved by the local animal care committee.

\section{Adhesion Assay \\ Untreated tissue culture plates (48 wells/plate) were covered with vitronectin at $10 \mu \mathrm{g} / \mathrm{ml}$ and incubated overnight at $4{ }^{\circ} \mathrm{C}$. Blocking for unspecific binding was with $0.5 \%$ BSA in PBS for $30 \mathrm{~min}$ at room temperature. After washing with PBS, the cells (50K/well) were added in adhesion buffer and the uPA peptide 1-48 added at $25 \mu \mathrm{g} / \mathrm{ml}$. Incubation was for $1 \mathrm{~h}$ at $37^{\circ} \mathrm{C}$. The detached cells and supernatant were removed and the wells gently washed three times with adhesion buffer. The cells were then fixed in crystal violet and air dried. The fixed cells were dissolved in methanol and OD 595 was determined on a plate reader.}

\section{Migration Assay}

Cell migration was performed in Transwell plates containing $12 \mu \mathrm{m}$ pore size filters (Costar, Cambridge, MA, USA). The upper side of the filter was coated with vitronectin $1 \mu \mathrm{g} / \mathrm{ml}$ and the lower side with vitronectin $10 \mu \mathrm{g} / \mathrm{ml}$. After incubation for $1 \mathrm{~h}$ at $37^{\circ} \mathrm{C}$, the filters were treated with $1 \%$ heat-treated BSA in PBS and then washed twice with PBS. Cells suspended in serum-free medium containing $25 \mu \mathrm{g} /$ $\mathrm{ml}$ of the PEG-uPA1-48 peptide or ATF $(0.3 \mu \mathrm{g} / \mathrm{ml})$ or unspecific mouse IgG were placed in the upper chamber $\left(5 \times 10^{4}\right.$ cells/chamber $)$ and incubated at $37^{\circ} \mathrm{C}$ in a humidified atmosphere of $5 \% \mathrm{CO}_{2}$ in air. After $20 \mathrm{~h}$, the cells on the upper side of the filter were removed with a cotton swab, and cells on the lower side of the filter were fixed and stained with Diff-Quik Stain Set (Dade Behring, Newark, DE, USA). The cells on the lower side of the filter were counted in nine randomly selected fields using $\times 20$ objective.

\section{Receptor-bound uPA}

Human BCEC were grown for $24 \mathrm{~h}\left(5 \times 10^{5}\right.$ cells/well in six-well plates), then washed twice with PBS and incubated with $4 \mathrm{ml}$ serum-free RPMI medium containing either PEGh1-48 $(25 \mu \mathrm{g} / \mathrm{ml})$ or ATF $(0.3 \mu \mathrm{g} / \mathrm{ml})$ or the solvent (PBS) alone (=controls). After $18 \mathrm{~h}$ incubation at $37^{\circ} \mathrm{C}$, the supernatants were harvested for determination of secreted uPA and the cultures were washed twice with ice-cold PBS. The cultures were then incubated for $3 \mathrm{~min}$ with $50 \mathrm{mM}$ glycine-HCl buffer ( $\mathrm{pH}$ 3.0) followed by neutralization with $0.5 \mathrm{M}$ Tris- $\mathrm{HCl}$ buffer $(\mathrm{pH} 7.8)$ as previously described. ${ }^{25}$ The eluates $(20 \mu \mathrm{l})$, cleared from cell debris by centrifugation, were subjected to sodium dodecyl sulfate polyacrylamide gel electro- phoresis and underlayed with a regular fibrinagarose indicator gel. ${ }^{25}$ Clear zones of lysis, indicating presence of active uPA, were identified after $6 \mathrm{~h}$ of incubation at $37^{\circ} \mathrm{C}$.

\section{Endothelial Tube Formation Assay}

Matrigel (Becton Dickinson) was added (150 $\mu \mathrm{l})$ to each well of a 48-well plate and allowed to polymerize for $1 \mathrm{~h}$ at $37^{\circ} \mathrm{C}$. A suspension of $10^{4}$ cells in $200 \mu$ l growth medium ${ }^{22}$ was then added into each coated well containing either $50 \mu \mathrm{g} / \mathrm{ml}$ of mouse or human pegylated uPA1-48 or ATF $(0.3 \mu \mathrm{g} / \mathrm{ml})$ or an unspecific immunoglobulin. Cells were incubated for $48 \mathrm{~h}$ at $37^{\circ} \mathrm{C}$, viewed using a Nikon TMS microscope (Nikon, Tokyo, Japan) and photographed using Matrox Inspector Software. The number of tubes was counted in four different fields of triplicate wells and the experiment repeated three times. Data are given in mean with s.d.

\section{Immunohistochemistry}

Paraffin-embedded tumors were cut into 5 - $\mu$ m-thick sections, deparaffinized in xylene, and hydrated in distilled water. All tissue sections were then treated with methanol containing $3 \%$ hydrogen peroxide, followed by blocking with $2 \%$ goat serum in PBS. For UPAR immunohistochemical assays, the sections were treated for $15 \mathrm{~min}$ with $0.1 \%$ trypsin (w/v) in $0.1 \% \mathrm{CaCl}_{2}(\mathrm{w} / \mathrm{v})$ in $0.1 \mathrm{~mol} / \mathrm{l}$ Tris/HCl, $\mathrm{pH}$ 7.8 , at $37^{\circ} \mathrm{C}^{26}{ }^{26}$ For CD31 staining, the sections were heated to $89^{\circ} \mathrm{C}$ for $10 \mathrm{~min}$ in sodium citrate buffer $\mathrm{pH}$ 6.0, and then allowed to cool down to room temperature over $20 \mathrm{~min}$. Ki-67 staining was performed with the sections immersed in $0.1 \mathrm{~mol} / \mathrm{l}$ citrate buffer, $\mathrm{pH}$ 6.0, and heated in a microwave oven to $97^{\circ} \mathrm{C}$ for $10 \mathrm{~min}$ and allowed to cool slowly to room temperature for antigen retrieval. Dilutions for the primary antibodies were for murine uPAR 1:25, human UPAR 1:100, murine CD 31 1:50, and human Ki-67 1:50. Primary antibody solutions were applied overnight at $4^{\circ} \mathrm{C}$, the slides were then washed with PBS and the signals were detected with DAKO LSAB ${ }^{\mathbb{R}} 2$ system, alkaline phosphatase (DAKO Corp., Carpinteria, CA, USA) for uPAR staining and with the histostain ${ }^{\mathrm{TM}}$-plus Kits (Zymed Labs, San Francisco, CA, USA) for CD31 and Ki-67 staining, following the manufacturer's instructions. All slides were counterstained with hematoxylin. PBS instead of primary antibodies was used for negative controls.

Blood-vessel density was quantified by counting the number of vessels per unit area across the entire tumor. ${ }^{27}$ The Ki-67 antigen labeling index (Ki-67 LI) was determined by counting a total of at least 1000 neoplastic nuclei subdivided in 10 fields chosen randomly at $\times 400$ magnification. ${ }^{28}$ 


\section{TUNEL Assay}

Apoptotic cells in tumor tissue were detected with the In situ Apoptosis Detection Kit (Roche Diagnostics, Indianapolis, IN, USA) according to the manufacturer's instructions. Briefly, $5 \mu \mathrm{m}$ tissue sections were mounted on glass slides treated with poly-L-lysine, deparaffinized, hydrated, and treated for $10 \mathrm{~min}$ at $37^{\circ} \mathrm{C}$ with proteinase $\mathrm{K}(20 \mu \mathrm{g} / \mathrm{ml}$ $10 \mathrm{mM}$ Tris-HCl buffer, $\mathrm{pH}$ 7.4). Slides were rinsed twice with PBS and then incubated with $50 \mu \mathrm{l}$ TUNEL reaction mixture ( $450 \mu \mathrm{l}$ nucleotide mixture containing fluoresceinated deoxy-UTP in reaction buffer plus $50 \mu$ l enzyme terminal deoxynucleotidyl transferase from calf thymus). After washing with PBS, slides were incubated for $30 \mathrm{~min}$ at room temperature in a humidified chamber with antifluorescein antibody conjugated to alkaline phosphatase, followed by $100 \mu \mathrm{l}$ substrate solution (fast red)/slide. Slides were then incubated at room temperature until suitable staining developed. Counterstaining was with hematoxylin. Negative controls were performed with Label Solution (without terminal transferase) instead of TUNEL reaction mixture. TUNEL-positive cells were counted at $\times 400$ magnification. The apoptotic index was determined by counting a total of at least 1000 neoplastic nuclei subdivided into 10 randomly chosen fields, as previously described. ${ }^{29}$

\section{FACS Analysis for Mouse and Human uPAR}

Adherent cells were dissociated into a single cell suspension with cell dissociation buffer (Gibco/ BRL, Gaithersburg MD, USA). After washing in PBS, the cells were incubated for $30 \mathrm{~min}$ on ice with optimal concentrations of either primary antibody or isotype control (Coulter, Raritan, NJ, USA). The primary antibodies were as under immunohistochemistry above at the same concentrations. After washing, the cells were incubated with secondary goat anti-mouse IGg conjugated with FITC, washed, and analyzed in a FACS Calibur flow cytometer (Becton-Dickinson, San Jose, CA, USA). In all, 10000 events were analyzed. The number of positively stained cells was determined by electronic subtraction of background staining of the cells stained with the isotype control antibody.

\section{Statistical Analysis}

Separate analyses of variance (ANOVA) were performed to investigate the effects of PEGh1-48 and PEGhm1-48 and/or human ATF on migration by human and murine endothelial cells, respectively, and the effect of PEGh1-48 on brain tumor cell migration. Three experiments were carried out in triplicate in each study. The overall F-test was based on the mean square (MS) due to the treatment effect divided by the MS due to experiment-to-experiment variability. The least significant difference (LSD) method was used for the pairwise comparisons once the overall F-test was significant at the 0.05 level. Means and $95 \%$ confidence intervals (CIs) were calculated based on the log-transformed data and then transformed back to the original scale.

To test the effects of PEGh1-48 and PEGhm1-48 on survival, two experiments were performed on the U87MG cell line. There were four treatment groups for each experiment; control, PEGh1-48, PEGhm1-48, and combined treatment. Kaplan-Meier plots and the log-rank test were used to compare survival time according to the treatment groups, with the log-rank test being based on the stratification by experiment. Pairwise comparison of the treatment groups was also performed using the stratified log-rank test for U87MG. Data from mice that were killed for unrelated and unknown causes or for a specific study question were censored at the date of killing. Tumor sizes measured at the time of death in different treatment groups were also compared using ANOVA. The LSD method was used for the pairwise comparisons, once the overall F-test was significant at the 0.05 level.

Analyses of variances were performed to compare apoptosis (TUNEL), cell proliferation (Ki-67), and neovascularization (CD31) among the control group and the groups treated with PEGh1-48 and PEGhm1-48 and the combination. There were five mice in each treatment group. For each tumor, 10 different fields were chosen and the positive cells or vessels were counted. The analysis on CD31 was based on the log-transformed data. The models all included PEGh1-48 (presence or absence), PEGhm1-48 (presence or absence), and the interaction between PEGh1-48 and PEGhm1-48. The overall F-test was based on the MS due to the treatment effect divided by the MS due to mouse-to-mouse variability. The LSD method was used for the pairwise comparisons once the overall F-test was significant at the 0.05 level. Means and 95\% CIs of CD31 were calculated based on the log-transformed data and then transformed back to the original scale.

\section{Results}

\section{Characterization of Pegylated uPA1-48 Proteins}

The binding of the serine protease, urokinase, to the cell surface is mediated by the GPI-linked protein, UPAR, in an interaction that is quantitatively accounted for by the growth factor domain of uPA, uPA1-48. ${ }^{20,30}$ This interaction shows significant species specificity for both the human and murine uPARs. ${ }^{8,19}$ We have previously reported the conversion of the human growth factor domain, h1-48, to a high-affinity ligand of the murine uPAR, hm1-48, by site-directed mutagenesis. ${ }^{8}$ In order to generate ligands for both receptors with improved in vivo half-lives, we modified the recombinant yeastderived growth factor domains selectively at their 
amino termini with a $20 \mathrm{kDa}$ polyethylene glycol moiety using the method of Gaertner and Offord ${ }^{21}$ as described in Materials and methods. Subsequent analysis showed that both PEGh1-48 and PEGhm1-48 have Ki's of 1-2 $\mathrm{nM}$ in receptor-binding assays with the human and murine uPAR, respectively. The addition of the PEG moiety increased the half-life of PEGh1-48 more than 10-fold in mice after i.v. administration from less than 10 to $130 \mathrm{~min}$, as described in Materials and methods and previously. ${ }^{31}$

\section{In Vitro Effects of Pegylated Proteins on Endothelial Cells}

In addition to expression by tumor cells, the uPA system is also expressed on activated endothelial cells in a variety of settings, and catalytically inactive UPAR ligands have been shown to reduce endothelial cell tube formation in vitro and angiogenesis in vivo. ${ }^{3,26,32}$ Previously, inhibition of tube formation had been reported for both anti-uPAR antibodies with human endothelial cells ${ }^{26}$ and murine growth factor domain-IgG fusion proteins with murine brain endothelial cells in fibrin gels. ${ }^{3}$ To investigate whether the pegylated uPA1-48 peptides affect endothelial tube formation, we tested both human and murine endothelial cells in a Matrigel tube formation assay. As illustrated in Figure 1 and quantified in Table 1, each peptide significantly reduced tube formation on its speciesspecific cells. In addition, we examined the effects of the pegylated peptides on vitronectin adhesion and migration of human and murine endothelial cells and brain tumor cells. Adhesion to vitronectin was not affected by the pegylated peptides for any of the cells tested. In contrast, the pegylated peptides significantly inhibited endothelial cell migration on a vitronectin gradient $(P<0.0001$ for both mouse and human brain endothelial cells). The PEGh1-48 also efficiently suppressed human ATF-stimulated migration (Figure 2a). Unmodified huPA1-48
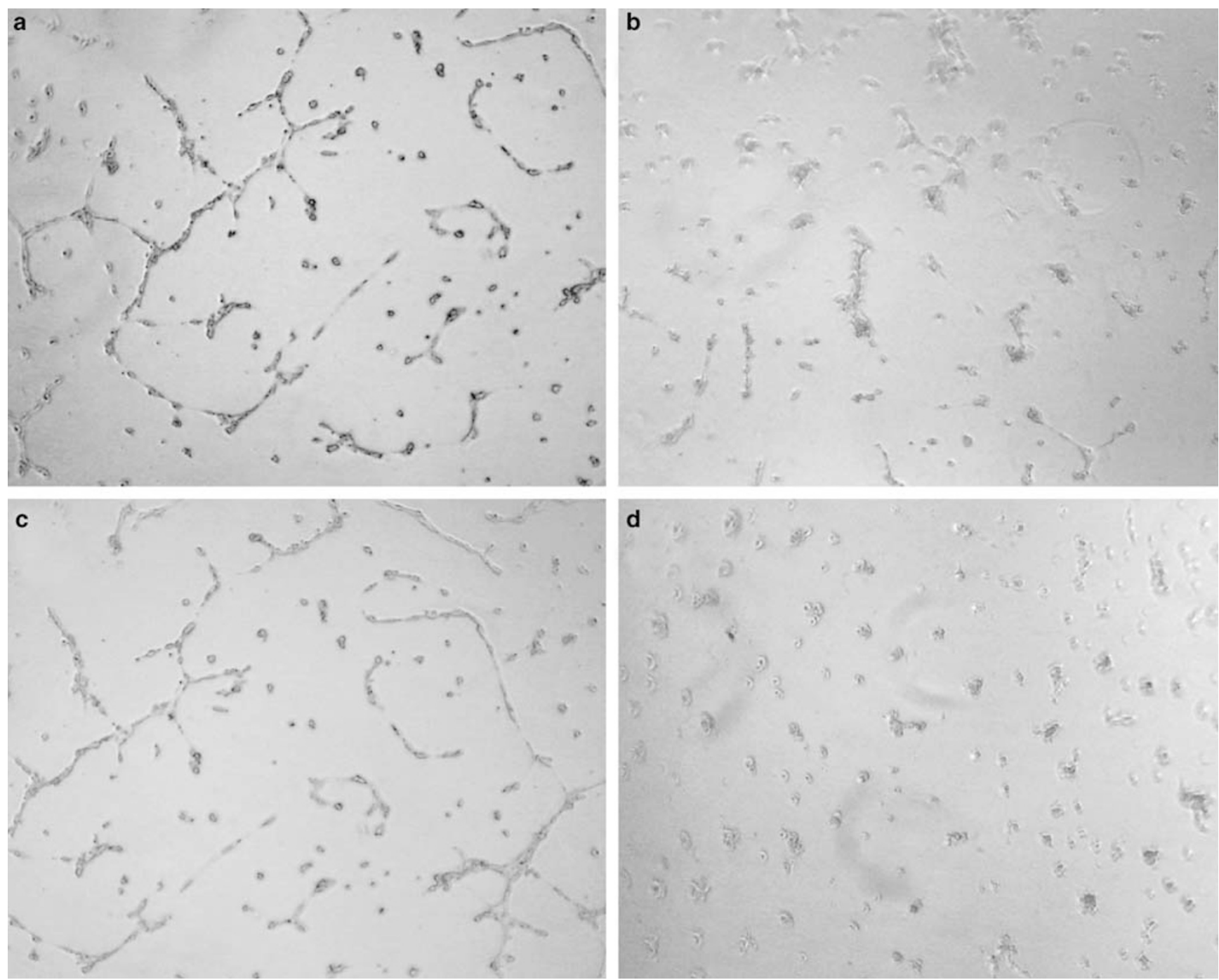

Figure 1 Tube formation by human and murine BCEC in the presence or absence of PEG-uPA1-48. Human BCEC control (a) and treated with PEGh1-48 (b); mouse BCEC control (c) and treated with PEGhm1-48 (d). Pictures were taken after 48 hr incubation at $\times 40$. 
Table 1 Endothelial cell tube formation in Matrigel in the presence or absence of PEGh1-48 and PEGhm1-48, respectively

\begin{tabular}{lccrr}
\hline Cells & Control & PEGh1-48 & P-value & PEGhm1-48 \\
\hline Human BCEC & $41.2 \pm 13.1$ & $10.8 \pm 5.5$ & 0.013 & $48.3 \pm 2.8$ \\
Mouse BCEC & $51.0 \pm 16.1$ & $53.5 \pm 3.8$ & 0.628 & $7.5 \pm 4.2$ \\
\hline
\end{tabular}

Cells were plated on Matrigel as outlined in Materials and methods and the tubes counted after $48 \mathrm{~h}$. Control=cultures treated with unspecific mouse IgG, $P$-values are in comparison to control cultures.
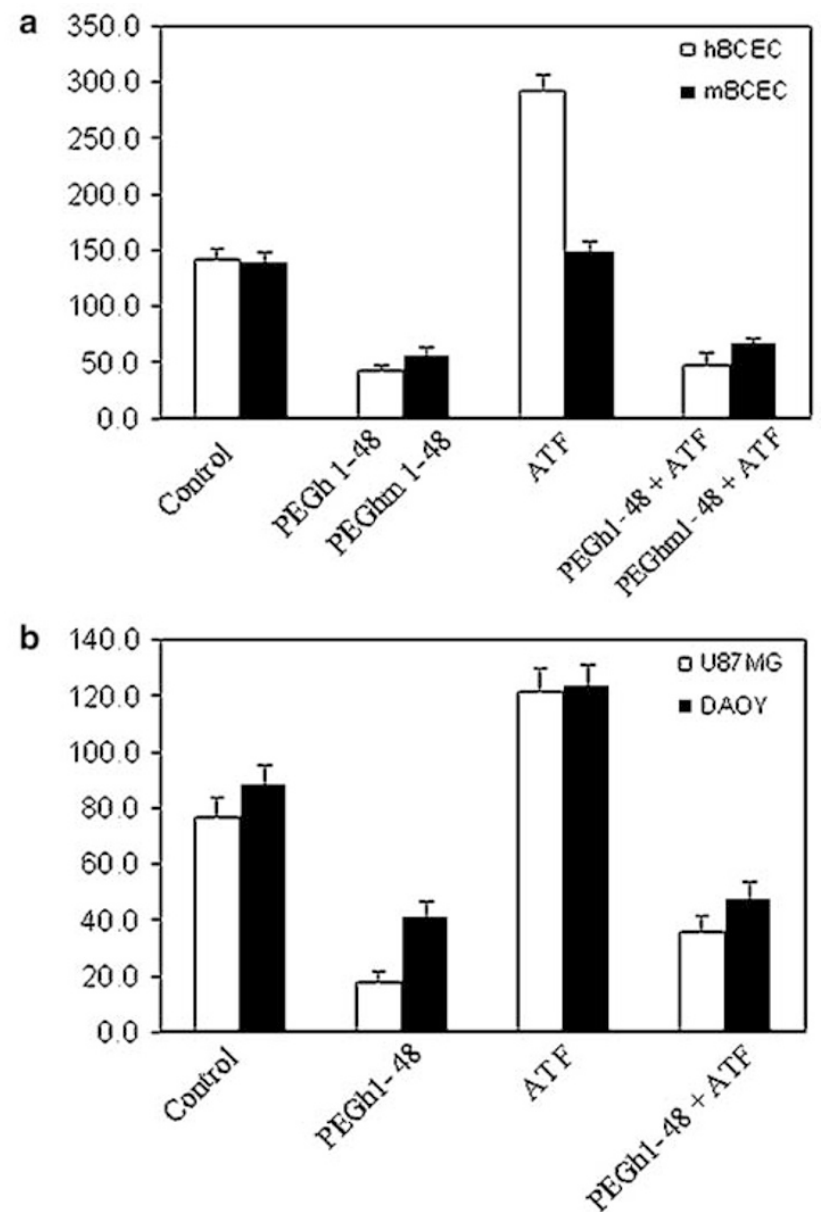

Figure 2 Cell migration on a vitronectin gradient in the presence or absence of PEG1-48 and/or human ATF. Experimental conditions were as described in Materials and methods. Numbers of migrating cells/high-power field are given as mean with s.d. of triplicate cultures of two experiments. $\mathbf{a}=$ Human (open bars) and mouse BCEC (black bars): Control=PBS; exposure to speciesspecific PEGh1-48 and PEGhm1-48; exposure to human ATF (only human BCEC respond with increased migration) and exposure to species-specific PEG 1-48 and human ATF (inhibitory effect of PEG 1-48 prevails in both conditions). $\mathbf{b}=$ brain tumor cells (U87MG = open and DAOY cells = black bars). Control $=$ PBS, exposure to PEGh1-48 or human ATF or both combined (PEGh$1-48+\mathrm{ATF})$.

decreased the migration of human brain endothelial cells, showing that pegylation did not affect the antimigratory activity (data not shown). No murine ATF was available for these studies, and human ATF did not change the migration of mouse brain endothelial cells, supporting the species specificity

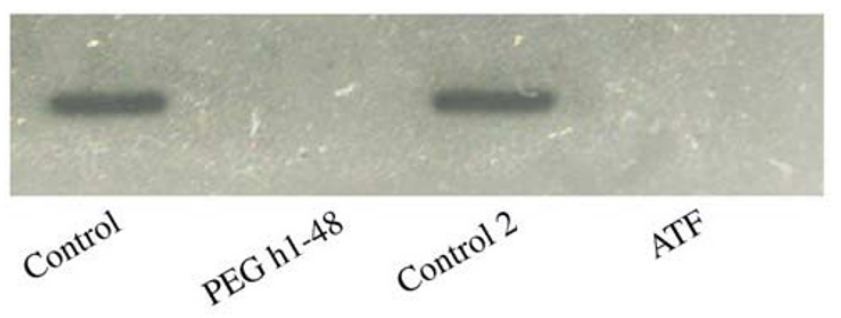

Figure 3 Influence of PEGh1-48 and ATF, respectively, on receptor bound uPA on human brain capillary cells. Eluates from human brain capillary cell cultures were separated by SDS-PAGE as described in Materials and methods. uPA activity was detected as zones of lysis (black) on a regular fibrin indicator gel.

of these peptides. PEG hm1-48 also did not alter the migration of the human brain endothelial cells, supporting its species-specific action (data not shown). A similar inhibitory effect on migration of the human-specific pegylated peptide was observed with the brain tumor cells (Figure 2b). Exposure of human BCEC to either PEGh1-48 or ATF resulted in the absence of receptor-bound uPA activity, demonstrating the prevention of uPA binding to its receptor by either compound (Figure 3). A similar absence of mouse uPA was detected in murine brain capillary cells when exposed to PEGhm1-48 (data not shown). Concomitant with the decrease of cell surface-bound uPA activity, the activity of secreted uPA increased in the supernatant (data not shown).

\section{In Vivo Treatment of Brain Tumors with Pegylated Proteins}

We then tested the effect by subcutaneous administration of PEGh1-48 and PEGhm1-48 alone and in combination in the orthotopic tumor model with U87MG cells implanted in the brains of nude mice. The effects of the peptides, administered twice weekly starting on day 4 after tumor implantation, on tumor size and survival are shown in Table 2 and Figure 4. All control animals were dead by day 75 . PEGh1-48 treatment shows a slight increase in survival $(P=0.019)$, but both treatments with PEGhm1-48 alone $(P=0.009)$ and in combination $(P=0.002)$ show a significant and marked increase in survival. It should be noted that the doses of both PEGh1-48 and PEGhm1-48 in the combination study were reduced by three-fold, making the combination result even more significant.

The increased survival time is also manifested in the average tumor sizes (Table 2). While the control 
Table 2 Average tumor diameters of the different treatment groups of U87MG tumors

\begin{tabular}{lccr}
\hline Treatment & Mean & $95 \%$ CI & P-value \\
\hline Control & 5.31 & $4.30-6.32$ & \\
PEGh1-48 & 3.29 & $2.31-4.26$ & 0.006 \\
PEGhm1-48 & 2.39 & $1.41-3.36$ & $<0.001$ \\
Both combined & 2.00 & $1.06-2.94$ & $<0.001$
\end{tabular}

Brains were removed at the time of sacrifice, fixed in $10 \%$ buffered formalin and embedded in paraffin. Serial sections were obtained, stained with H\&E and the biggest diameter of the tumors determined. Results are given in means and $95 \%$ CI. $P$-values are in comparison to the control tumors.

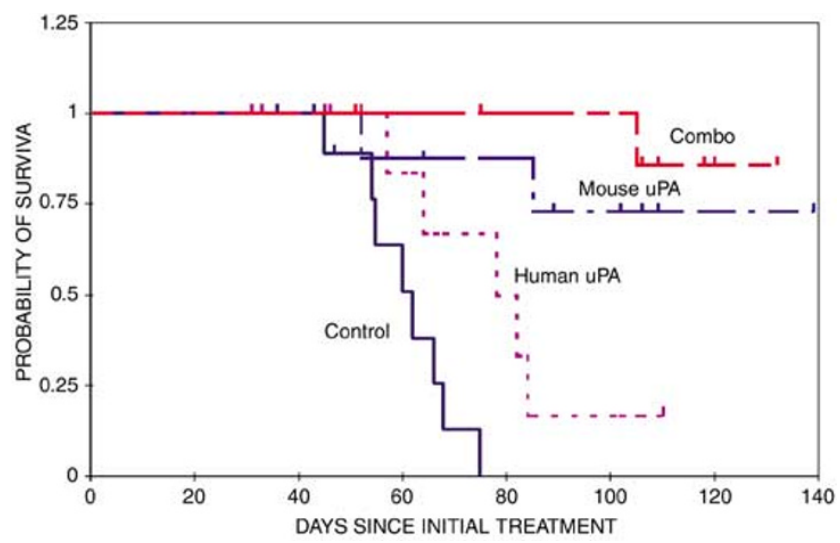

Figure 4 Survival of mice injected with U87MG brain tumors. Treatment was initiated on day 4 and continued twice weekly s.c. until mice had to be sacrificed due to tumor progression or termination of the experiment. Each study arm had 10 animals.

tumors had a mean diameter of $5.31 \mathrm{~mm}$ (95\% CI $4.30-6.32 \mathrm{~mm}$ ), it decreased to $3.29 \mathrm{~mm}$ in tumors treated with PEGh1-48 $(P=0.006)$ and $2.39 \mathrm{~mm}$ in those treated with PEGhm1-48 $(P<0.001)$. Furthermore, animals exposed to the combined treatment had an average tumor diameter of $2.0 \mathrm{~mm}$ with a $P$-value of $<0.001$ when compared to control tumors.

\section{Analysis of Tumor Growth Inhibition}

It has been reported that glioblastoma cell lines express high levels of uPA and uPAR. $^{33}$ We first tested the U87MG cells used in this study in vitro by staining with a monoclonal antibody to human UPAR and showing by FACS analysis that greater than $90 \%$ of the cells were positive for uPAR expression. In order to ascertain that uPAR is also expressed in vivo, control tumor slices from U87MG tumors were stained with a monoclonal antibody to human UPAR and a rabbit antibody to murine uPAR. The results of this study are shown in Figure 5a, where it can be seen that tumor cells are labeled with the human uPAR antibody, consistent with the known species specificity of the antibody. The murine UPAR antibody intensely stains the tumor vasculature (Figure 5b), consistent with other reports that UPAR expression is upregulated in endothelial cells during tissue remodeling, and is expressed in human glioma tumor vasculature. ${ }^{34-36}$ Indeed, FACS analysis demonstrated high levels of UPAR in murine BCEC as has been shown previously for human brain microvascular cells. ${ }^{37} \mathrm{~A}$ comparison of the level of neovascularization, as judged by CD31 expression, for the group receiving combined treatment with PEGh1-48 and PEGhm1-48 and control groups, was measured by immunohistochemistry and a representative example is shown in Figure 5c and d. Clearly, the extent of vascularity of the treated tumors is qualitatively decreased from those of the controls. To further analyze the effects of the uPAR antagonists on in vivo tumor growth and progression, tumors from all four treatment groups were stained with Masson's Trichrome for collagen deposition (Figure 6). Increased collagen deposition is seen in all the treated groups vs control, with the most pronounced effects in the PEG hm1-48 and combination-treated tumors.

A quantitative analysis of tumor cell proliferation (Ki-67), apoptosis (TUNEL), and neovascularization (vessel counts) was then undertaken to further investigate the tumors observed in the treated groups, with the results shown in Table 3 . These data show that the blockade of both tumor and stromal uPAR by either PEGh1-48 or PEGhm1-48 increased tumor cell apoptosis $(P<0.001$ for both human and mouse) and reduced neovascularization $(P<0.001$ for both human and mouse), although their effects on tumor cell proliferation did not quite attain statistical significance $(P=0.070$ for PEGh1-48, $P=0.053$ for PEGhm1-48). Furthermore, PEGh1-48 and PEGhm1-48 had a significant synergistic effect on both tumor cell apoptosis and neovascularization $(P \leq 0.001$ and $P=0.030$ for the interaction terms, respectively).

\section{Discussion}

The uPA system has been implicated in a number of processes involved in tumor progression, including extracellular matrix degradation, tumor cell invasion, metastasis, and angiogenesis (reviewed in Andreasen et al. ${ }^{1,2}$ A large body of studies indicate that UPA and its cognate serpin inhibitor, PAI-1, are independent prognostic indicators in human cancers. ${ }^{38}$ Studies using immunohistochemistry and in situ hybridization have shown that UPA and UPAR are sometimes expressed by different cell types within tumors, and that especially in breast carcinomas, myofibroblastic stromal cells are often the source of uPA. ${ }^{13,39}$ The role of stromal uPA has been investigated in several studies using uPA-deficient mice, with different results being obtained in different tumor models. In one case $\mathrm{uPA}^{-} /^{-}$and immunodeficient mice were constructed and the growth of MDA-MB435 tumors was investigated..$^{14}$ 

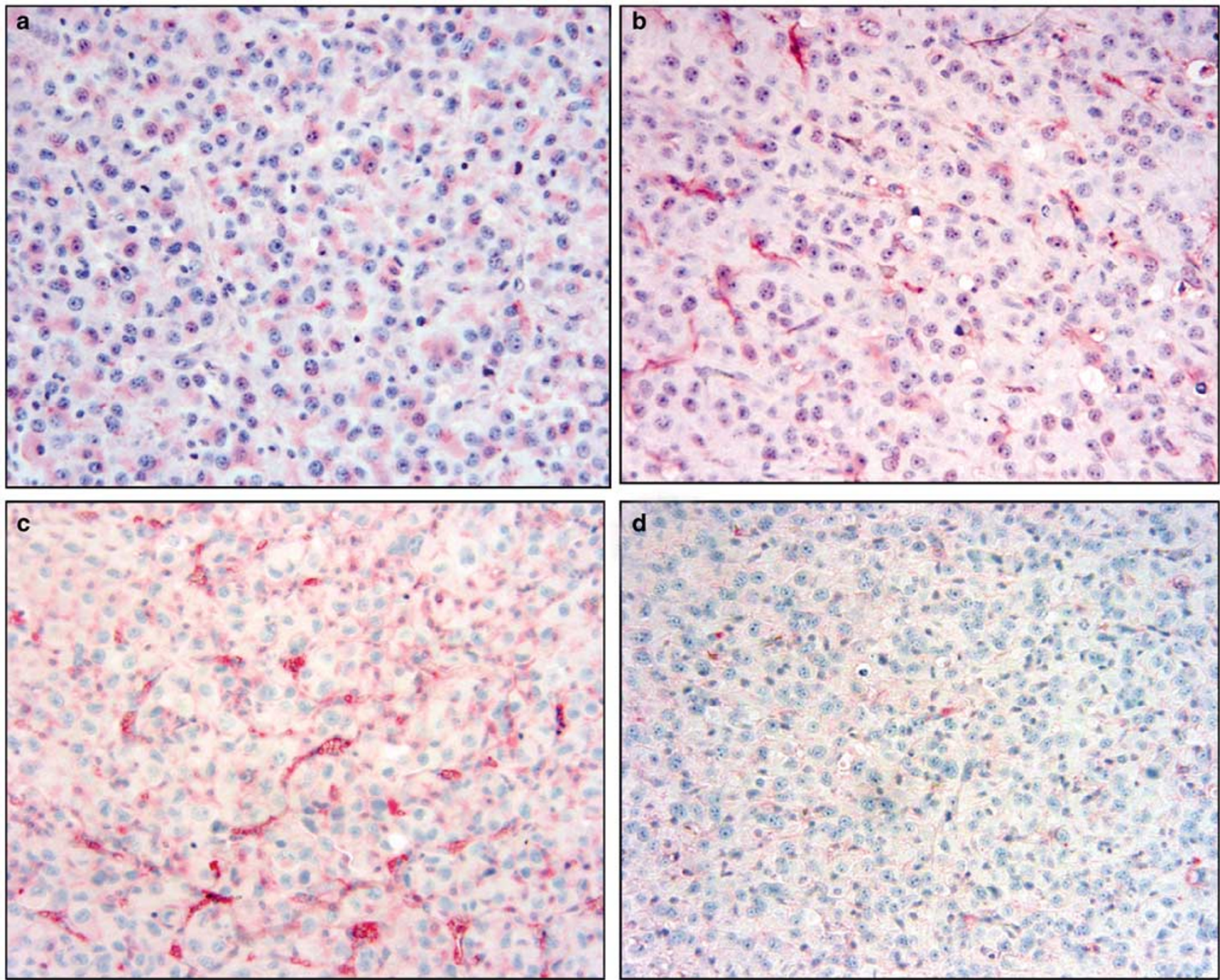

Figure 5 Staining of tumor tissue for uPAR (a and $\mathbf{b}$ ) and CD 31 (c and d) in U87MG tumors. A control tumor was used for staining human uPAR (tumor cells) using the mouse monoclonal antibody 3936 (a) and a rabbit anti-mouse antibody for mouse endothelial cells (b). Magnification is $\times 200$. CD31 staining for murine vascular endothelial cells was performed with the rat anti-mouse monoclonal antibody MEC 13.3 in a control tumor (c) and a tumor treated with both PEG-uPA1-48 peptides (d). Conditions were as described in Material and methods. Magnification is $\times 200$.

Tumor growth was retarded in the $\mathrm{uPA}^{-/^{-}}$mice, but neither invasion nor angiogenesis was significantly affected. In a second study, Gutierrez et $a l^{15}$ used a syngeneic tumor model of T241 fibrosarcoma in $\mathrm{uPA}^{-} /^{-}$and PAI-1 ${ }^{-{ }^{-}}$mice. In this case, both tumor growth and metastasis were reduced in both knockout mice, and tumor cell proliferation was decreased and apoptosis increased, whereas tumor neovascularization was only affected in the $\mathrm{PAI}-1^{-/^{-}}$mice. Other studies showed modest effects of uPA deficiency on tumor growth and angiogenesis. ${ }^{40,41}$

Another approach taken was to use a defective adenovirus to express a murine ATF systemically, which prolongs mouse survival and prevents liver metastasis in a colon carcinoma model. ${ }^{32}$ In this study, a significant reduction in tumor angiogenesis and increase in apoptotic index was observed, in contrast to that seen in the $\mathrm{uPA}^{-} /^{-}$mice. A related study examined the effect of a murine uPA growth factor domain-IgG fusion protein on tumor growth and angiogenesis in a syngeneic B16 melanoma mouse model. ${ }^{3}$ In this case, significant reductions in both tumor size and angiogenesis were observed. Although different tumor models have been used in all of these studies, the results on angiogenesis, in particular, suggest that UPA deficiency and saturation of UPAR with a noncatalytically active ligand can cause different effects, perhaps on uPAR signaling. ${ }^{42}$

Gliomas are known to express high levels of uPA and UPAR both in vitro and in vivo, and are also highly angiogenic. ${ }^{43}$ Previous studies on U87MG cells have shown that an $\alpha \mathrm{v}$-integrin antagonist, EMD 121794, can largely prevent tumor growth, but only when the cells are implanted orthotopically, and that this effect is mediated through both 

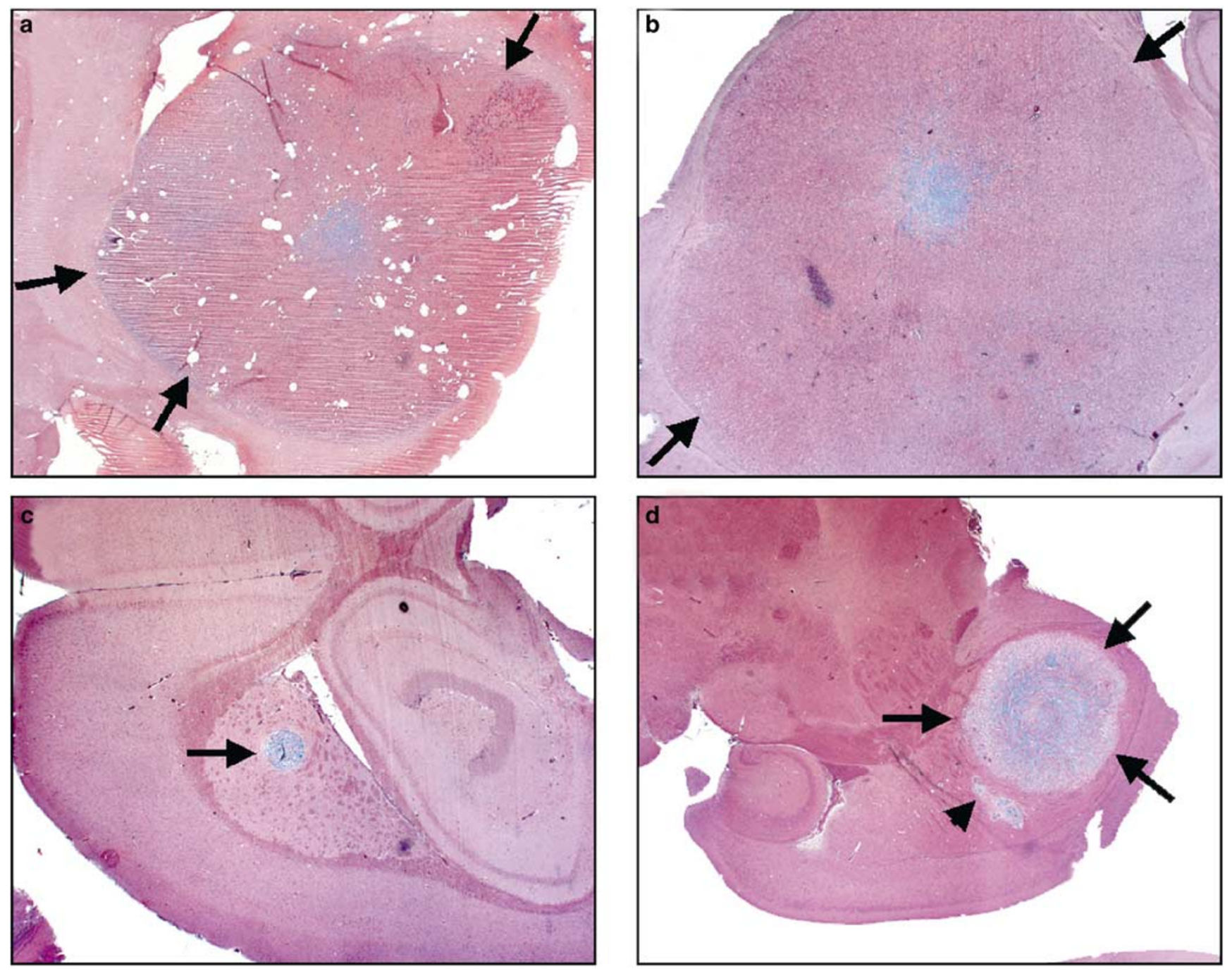

Figure 6 Masson's Trichrome staining of U87MG tumors with (a) Control tumor (day 64), (b) Tumor treated with human uPA peptide (day 82), (c) Tumor treated with mouse peptide (day 47), and (d) Both peptides used for treatment (day 136). Collagenous material stains in blue. Magnification is $\times 200$.

Table 3 Average value of TUNEL-positive cells, the Ki-67 labeling index, and the microvascular density MVD (CD31-positive structures) in the different treatment groups of U87MG tumors

\begin{tabular}{lcccc}
\hline & TUNEL & P-value & Ki-67 & MVD \\
\hline Control & $0.92(0.28-1.56)$ & & $22.5(11.8-33.1)$ & $196.4(172.4-225.9)$ \\
PEGh1-48 & $2.58(1.94-3.22)$ & $<0.001$ & $18.5(9.7-27.3)$ & $104.6(90.9-119.1)$ \\
PEGhm1-48 & $3.15(2.35-3.95)$ & $<0.001$ & $18.3(8.5-28.1)$ & $86.5(73.0-103.5)$ \\
Both combined & $8.42(7.75-9.09)$ & $<0.001$ & $7.3(1.5-16.1)$ & $32.5(27.9-37.7)$ \\
\hline
\end{tabular}

Results are given as mean and $95 \%$ CI for five tumors for each group with 10 microscopic fields evaluated at $\times 400$. $P$-values are in comparison to control. The Ki-67 labeling index did not read significant differences.

endothelial cells as well as $\alpha \mathrm{v}$ expressing tumor cells. ${ }^{24,44}$ In addition, other workers have used defective adenoviruses encoding antisense constructs to UPAR and have shown that the resulting decreased uPAR expression suppressed tumor cell invasion and tumor growth. ${ }^{18}$

In this study, we have constructed catalytically inactive UPAR ligands suitable for in vivo adminis- tration by covalent modification of uPA growth factor domains with single-amino-terminal polyethylene glycol moieties. Using the known structure-activity relationship for species specificity in uPAR binding, human uPA1-48 and a mutant form were constructed and expressed having high-affinity binding for either the human or murine uPAR, respectively. ${ }^{8,19}$ Modification of these molecules 
with the PEG moiety had a small effect on binding affinity, whereas the in vivo half-life was increased more than 10-fold. We first tested the effects of these molecules on the migration on vitronectin gradients of human and murine capillary endothelial cells, as well as the tumor cells used in this study. As has been shown previously for the brain tumor cells used in this study, ATF stimulates migration, ${ }^{33}$ whereas, surprisingly, addition of PEGh1-48 inhibits the ATF-mediated stimulation of migration. Prior work in the human UPAR system has shown that ATF and GFD bind to UPAR with the same affinity. This indicates an additional role for the kringle domain of UPA in UPAR-mediated migration effects, as has been seen in migration of vascular smooth muscle cells, ${ }^{45}$ or some unknown effect of the PEG moiety on uPAR signaling. Recent studies have suggested that the kringle domain of uPA is important for high-affinity binding of uPAR to vitronectin in vitro, mediated through uPAR dimerization. ${ }^{46}$ More importantly, the kringle domain of uPA has been shown to directly interact with the I-domain of $\alpha \mathrm{M} \beta 2$, to create trimolecular interactions between uPA, UPAR, and the integrin, with uPA as the bridging ligand. ${ }^{47}$ Our observations that the uPA1-48 peptides block ATF-stimulated migration for endothelial and tumor cells suggest that these phenomena are more widespread. The possibility that the uPA kringle domain may also interact with I-like domains of other integrins known to be important for glioma and endothelial cell proliferation should not be discounted, in the light of the recent elucidation of the structure of the $\alpha \mathrm{v} \beta 3$ integrin. ${ }^{48,49}$ More recently, it has been suggested that the uPA kringle stabilizes uPA binding to its receptor mediated by the amino-terminal growthfactor-like domain. ${ }^{50}$ Our data demonstrate that PEG 1-48 inhibits, like ATF, the binding of uPA to its receptor, although it is missing the kringle domain. The use of recombinant uPA1-48 rather than proteolytically derived uPA4-43 may account for this difference. Thus, under our test conditions, the growth-factor-like domain was sufficient to compete with native uPA for the binding to the receptor.

We then examined the effect of PEGh1-48 and PEGhm1-48 on both the murine (stromal) and human (tumor) receptors in an orthotopic brain tumor model using U87MG cells. A very significant effect on tumor growth and survival was observed in both the PEGhm1-48 and combination-treated groups, whereas the PEGh1-48 group had only a small, but still significant, increase in survival for the U87MG tumors. Although the survival difference between the PEGhm1-48 and combination groups is not significant, the effects on the tumor parameters of proliferation, apoptosis, and vascular density are highly significant. This effect appears to be primarily due to a reduction in the vascular density of the treated tumors (Figure 4) and is likely an antiangiogenic effect directly on the stromal endothelial cells, since PEGhm1-48 binds with greater than 100-fold selectivity to murine uPAR. The effect is particularly important because it suggests that the addition of a catalytically inactive UPAR ligand does not mimic the phenotype seen in the uPA knock-out studies described above, where no effect on tumor angiogenesis was observed. This may also reflect the differences between the tumor types used (breast carcinoma and fibrosarcoma vs glioma). Interestingly, in a breast carcinoma model, similar effects on tumor growth inhibition were seen with both host- and tumor- specific uPAR antagonists, suggesting that different tumor types rely on the stromal- and tumor- derived uPA systems to differing extents. ${ }^{8}$ This parallels what is seen in human tumors. ${ }^{2}$

The favorable response of brain tumors to treatment with angiogenesis inhibitors does not come as a surprise. The brain is protected from the influx of most drugs through the blood-brain barrier (BBB), which also explains the poor response of brain tumors to chemotherapeutic agents. ${ }^{51}$ The BBB may keep the angiogenesis inhibitor in the brain vasculature for prolonged times, resulting in an increased exposure of the endothelial cells. Also, death of endothelial cells induced by antiangiogenic agents allows for the decrease of the high interstitial pressure of brain tumors, opening up compressed novel tumor vessels and facilitating drug transfer through the now leaky tumor vasculature. ${ }^{52,53}$

The effects observed in this study on tumor cell apoptosis and proliferation are consistent with a marked antiangiogenic effect of the PEGhm1-48 molecule, especially in combination with PEGh1-48 treatment. This treatment also results in a dramatic increase in collagen deposition in the tumors, which may be due to connections between the regulation of the UPA system and especially PAI- 1 , and TGF- $\beta$, and also perhaps involving hepatocyte growth factor. The combined effect provides additional evidence that both the tumor cell and stromal cell uPA systems are important in tumor cell survival and progression. Possible molecular mechanisms for these effects include blockade of uPA: uPARmediated extracellular matrix degradation, which decreases endothelial cell invasion and reduces release of matrix-associated angiogenic factors by tumor cells. Alternatively, blockade of uPAR by a catalytically inactive peptide may result in lack of activation of metalloproteinases, resulting in accumulation of collagenous material as observed in our tumors, and as has been seen in uPA-deficient mice. Although we observed decreased binding of exogenous UPA to UPAR in the presence of the pegylated growth factor domains, we did not observe an alteration of MMP-2 and MMP-9 activities in vitro (data not shown). Another possibility is that blockage of uPAR may reduce the expression of $\alpha v$-integrins, which may alter adhesion and/or migration in vivo. ${ }^{54}$ In addition, modulation of effects on uPAR-integrin complexes that have been shown in other systems to be important in the 
balance between tumor cell proliferation and dormancy-may be involved in our model. ${ }^{11}$ The increasing recognition of the role of the uPA kringle domain in integrin-mediated processes and the antagonism of these effects by the uPA1-48 peptides is likely to play a significant part in the effects seen. ${ }^{47}$ Finally, inhibition of uPAR recycling by prevention of uPA:PAI-1 complex clearance via LRP and related receptors may play a role. ${ }^{55}$ Further dissection of these effects will be enabled by the availability of the immunodeficient uPA- knock-out mice, ${ }^{14}$ for example, by determining whether tumor growth is affected in these mice, and also whether treatment with PEGhm1-48 or related molecules still has an effect in the absence of host uPA. Such effects have been seen in a model of chemokine-induced leukocyte migration in uPA-deficient mice. ${ }^{16}$ Gyetko et $a l^{56}$ have observed that uPAR-deficient mice demonstrate impaired recruitment of leukocytes in response to infection and that such an altered response might affect tumor growth. Since leukocyte infiltration of xenotransplanted brain tumors is a very rare occurrence, and we have not observed any such infiltrates in our studies, such a mechanism is unlikely to be responsible for the slower tumor growth in our mice treated with these peptides. The results presented in this paper suggest strongly that the modulation of the uPA system may be valuable in the treatment of malignant gliomas.

\section{Acknowledgement}

This work was supported by grants from the NIH (CA 82989) and the TJ Martell Foundation to WEL and by Chiron Corporation, 4560 Horton St, Emeryville, CA 94608, USA.

\section{References}

1 Andreasen PA, Egelund R, Petersen HH. The plasminogen activation system in tumor growth, invasion, and metastasis. Cell Mol Life Sci 2000;57:25-40.

2 Andreasen PA, Kjoller L, Christensen L, et al. The urokinase-type plasminogen activator system in cancer metastasis: a review. Int J Cancer 1997;72:1-22.

3 Min HY, Doyle LV, Vitt CR, et al. Urokinase receptor antagonists inhibit angiogenesis and primary tumor growth in syngeneic mice. Cancer Res 1996;56: 2428-2433.

4 Janicke F, Prechtl A, Thomssen C, et al. Randomized adjuvant chemotherapy trial in high-risk, lymph nodenegative breast cancer patients identified by urokinasetype plasminogen activator and plasminogen activator inhibitor type 1. J Natl Cancer Inst 2001;93:913-920.

5 Foekens JA, Peters HA, Look MP, et al. The urokinase system of plasminogen activation and prognosis in 2780 breast cancer patients. Cancer Res 2000;60: 636-643.

6 Wei Y, Waltz DA, Rao N, et al. Identification of the urokinase receptor as an adhesion receptor for vitronectin. J Biol Chem 1994;269:32380-32388.
7 Wei Y, Lukashev M, Simon DI, et al. Regulation of integrin function by the urokinase receptor. Science 1996;273:1551-1555.

8 Tressler RJ, Pitot PA, Stratton JR, et al. Urokinase receptor antagonists: discovery and application to in vivo models of tumor growth. APMIS 1999;107: 168-173.

9 Li H, Lu H, Griscelli F, et al. Adenovirus-mediated delivery of a uPA/uPAR antagonist suppresses angiogenesis-dependent tumor growth and dissemination in mice. Gene Ther 1998;5:1105-1113.

$10 \mathrm{Yu} \mathrm{W,} \mathrm{Kim} \mathrm{J,} \mathrm{Ossowski} \mathrm{L.} \mathrm{Reduction} \mathrm{in} \mathrm{surface}$ urokinase receptor forces malignant cells into a protracted state of dormancy. J Cell Biol 1997;137: 767-777.

11 Aguirre Ghiso JA, Kovalski K, Ossowski L. Tumor dormancy induced by downregulation of urokinase receptor in human carcinoma involves integrin and MAPK signaling. J Cell Biol 1999;147:89-104.

12 Pyke C, Graem N, Ralfkiaer E, et al. Receptor for urokinase is present in tumor-associated macrophages in ductal breast carcinoma. Cancer Res 1993;53: 1911-1915.

13 Nielsen BS, Sehested M, Duun S, et al. Urokinase plasminogen activator is localized in stromal cells in ductal breast cancer. Lab Invest 2001;81:1485-1501.

14 Frandsen TL, Holst-Hansen C, Nielsen BS, et al. Direct evidence of the importance of stromal urokinase plasminogen activator (uPA) in the growth of an experimental human breast cancer using a combined uPA gene-disrupted and immunodeficient xenograft model. Cancer Res 2001;61:532-537.

15 Gutierrez LS, Schulman A, Brito-Robinson T, et al. Tumor development is retarded in mice lacking the gene for urokinase-type plasminogen activator or its inhibitor, plasminogen activator inhibitor-1. Cancer Res 2000;60:5839-5847.

16 Waltz DA, Fujita RM, Yang X, et al. Nonproteolytic role for the urokinase receptor in cellular migration in vivo. Am J Respir Cell Mol Biol 2000;22:316-322.

17 Mohanam S, Gladson CL, et al. Biological significance of the expression of urokinase-type plasminogen activator receptors (uPARs) in brain tumors. Front Biosci 1999;4:D178-D187.

18 Mohan PM, Chintala SK, Mohanam S, et al. Adenovirus-mediated delivery of antisense gene to urokinase-type plasminogen activator receptor suppresses glioma invasion and tumor growth. Cancer Res 1999;59:3369-3373.

19 Quax PH, Grimbergen JM, Lansink M, et al. Binding of human urokinase-type plasminogen activator to its receptor: residues involved in species specificity and binding. Arterioscler Thromb Vasc Biol 1998;18: 693-701.

20 Stratton-Thomas JR, Min HY, Kaufman SE, et al. Yeast expression and phagemid display of the human urokinase plasminogen activator epidermal growth factor-like domain. Protein Eng 1995;8:463-470.

21 Gaertner HF, Offord RE. Site-specific attachment of functionalized poly(ethylene glycol) to the amino terminus of proteins. Bioconjug Chem 1996;7:38-44.

22 Stins MF, Gilles F, Kim KS. Selective expression of adhesion molecules on human brain microvascular endothelial cells. J Neuroimmunol 1997;76: 81-90.

23 Goodson RJ, Doyle MV, Kaufman SE, et al. Highaffinity urokinase receptor antagonists identified with 
bacteriophage peptide display. Proc Natl Acad Sci USA 1994;91:7129-7133.

24 MacDonald TJ, Taga T, Shimada H, et al. Preferential susceptibility of brain tumors to the antiangiogenic effects of an alpha(v) integrin antagonist. Neurosurgery 2001;48:151-157.

25 Laug WE, Cao XR, T’Ang A, et al. Complex expression of the genes coding for plasminogen activators and their inhibitors in HeLa-smooth muscle cell hybrids. Cell Growth Differ 1992;3:191-197.

26 Kroon ME, Koolwijk $\mathrm{P}$, van Goor $\mathrm{H}$, et al. Role and localization of urokinase receptor in the formation of new microvascular structures in fibrin matrices. Am J Pathol 1999;154:1731-1742.

27 Reynolds LE, Wyder L, Lively JC, et al. Enhanced pathological angiogenesis in mice lacking beta3 integrin or beta3 and beta5 integrins. Nat Med 2002;8: 27-34.

28 Losa M, Barzaghi RL, Mortini P, et al. Determination of the proliferation and apoptotic index in adrenocorticotropin-secreting pituitary tumors: comparison between micro- and macroadenomas. Am J Pathol 2000; 156:245-251.

29 Losa M, Ciccarelli E, Mortini P, et al. Effects of octreotide treatment on the proliferation and apoptotic index of GH-secreting pituitary adenomas. J Clin Endocrinol Metab 2001;86:5194-5200.

30 Appella E, Robinson EA, Ullrich SJ, et al. The receptorbinding sequence of urokinase. A biological function for the growth-factor module of proteases. J Biol Chem 1987;262:4437-4440.

31 Drummond RJ, Rosenberg S. Method for increasing the serum half-life of a biologically active molecule. Patent: 6423685, 2002.

$32 \mathrm{Li} \mathrm{H}$, Griscelli F, Lindenmeyer F, et al. Systemic delivery of antiangiogenic adenovirus AdmATF induces liver resistance to metastasis and prolongs survival of mice. Hum Gene Ther 1999;10: 3045-3053.

33 MacDonald TJ, DeClerck YA, Laug WE. Urokinase induces receptor mediated brain tumor cell migration and invasion. J Neurooncol 1998;40:215-226.

34 Yamamoto M, Sawaya R, Mohanam S, et al. Expression and localization of urokinase-type plasminogen activator receptor in human gliomas. Cancer Res 1994;54: 5016-5020.

35 Solberg H, Ploug M, Hoyer-Hansen G, et al. The murine receptor for urokinase-type plasminogen activator is primarily expressed in tissues actively undergoing remodeling. J Histochem Cytochem 2001;49: 237-246.

36 Yamamoto M, Ikeda K, Ohshima K, et al. Expression and cellular localization of low-density lipoprotein receptor-related protein/alpha 2-macroglobulin receptor in human glioblastoma in vivo. Brain Tumor Pathol 1998;15:23-30.

37 Isogai C, Laug WE, Shimada $\mathrm{H}$, et al. Plasminogen activator inhibitor-1 promotes angiogenesis by stimulating endothelial cell migration toward fibronectin. Cancer Res 2001;61:5587-5594.

38 Look MP, van Putten WL, Duffy MJ, et al. Pooled analysis of prognostic impact of urokinase-type plasminogen activator and its inhibitor PAI-1 in 8377 breast cancer patients. J Natl Cancer Inst 2002;94: 116-128.
39 Nielsen BS, Sehested M, Timshel S, et al. Messenger RNA for urokinase plasminogen activator is expressed in myofibroblasts adjacent to cancer cells in human breast cancer. Lab Invest 1996;74:168-177.

40 Curino A, Mitola DJ, Aaronson $\mathrm{H}$, et al. Plasminogen promotes sarcoma growth and suppresses the accumulation of tumor-infiltrating macrophages. Oncogene 2002;21:8830-8842.

41 Sabapathy KT, Pepper MS, Kiefer F, et al. Polyoma middle T-induced vascular tumor formation: the role of the plasminogen activator/plasmin system. J Cell Biol 1997;137:953-963.

42 Blasi F, Carmeliet P. uPAR: a versatile signalling orchestrator. Nat Rev Mol Cell Biol 2002;3:932-943.

43 Zhang $\mathrm{X}, \mathrm{Bu} \mathrm{X}$, Zhen $\mathrm{H}$, et al. Expression and significance of urokinase-type plasminogen activator in human gliomas. Chin Med J (Engl) 2000;113: 802-804.

44 Taga T, Suzuki A, Gonzalez-Gomez I, et al. Alpha v-integrin antagonist EMD 121974 induces apoptosis in brain tumor cells growing on vitronectin and tenascin. Int J Cancer 2002;98:690-697.

45 Mukhina S, Stepanova V, Traktouev D, et al. The chemotactic action of urokinase on smooth muscle cells is dependent on its kringle domain. Characterization of interactions and contribution to chemotaxis. J Biol Chem 2000;275:16450-16458.

46 Sidenius N, Sier CF, Ullum H, et al. Serum level of soluble urokinase-type plasminogen activator receptor is a strong and independent predictor of survival in human immunodeficiency virus infection. Blood 2000;96:4091-4095.

47 Pluskota E, Soloviev DA, Plow EF. Convergence of the adhesive and fibrinolytic systems: recognition of urokinase by integrin alpha Mbeta 2 as well as by the urokinase receptor regulates cell adhesion and migration. Blood 2003;101:1582-1590.

48 Liddington RC, Ginsberg $\mathrm{MH}$. Integrin activation takes shape. J Cell Biol 2002;158:833-839.

49 Xiong JP, Stehle T, Diefenbach B, et al. Crystal structure of the extracellular segment of integrin alpha Vbeta3. Science 2001;294:339-345.

50 Bdeir K, Kuo A, Sachais BS, et al. The kringle stabilizes urokinase binding to the urokinase receptor. Blood 2003;102:3600-3608.

51 Pardridge WM. Drug and gene targeting to the brain with molecular Trojan horses. Nat Rev Drug Discov 2002;1:131-139.

52 Kerbel R, Folkman J. Clinical translation of angiogenesis inhibitors. Nat Rev Cancer 2002;2:727-739.

53 Jain RK. Normalizing tumor vasculature with antiangiogenic therapy: a new paradigm for combination therapy. Nat Med 2001;7:987-989.

54 Adachi Y, Chandrasekar N, Kin Y, et al. Suppression of glioma invasion and growth by adenovirus-mediated delivery of a bicistronic construct containing antisense $\mathrm{UPAR}$ and sense p16 gene sequences. Oncogene 2002;21:87-95.

55 Degryse B, Sier CF, Resnati M, et al. PAI-1 inhibits urokinase-induced chemotaxis by internalizing the urokinase receptor. FEBS Lett 2001;505:249-254.

56 Gyetko MR, Sud S, Kendall T, et al. Urokinase receptor-deficient mice have impaired neutrophil recruitment in response to pulmonary Pseudomonas aeruginosa infection. J Immunol 2000;165:1513-1519. 